\title{
Co-Infection with Neisseria gonorrhoeae, Chlamydia trachomatis and Trichomonas vaginalis in Symptomatic South African Men with Urethritis: Implications for Syndromic Management
}

\author{
M. De Jongh ${ }^{1}$, M. Le Roux ${ }^{1}$, A. Adam ${ }^{2}$, A.M. Caliendo ${ }^{3}$ and A.A. Hoosen ${ }^{4, *}$
}

${ }^{1}$ Department of Microbiological Pathology, University of Limpopo, Medunsa Campus, Ga-Rankuwa, Pretoria, 0204,
South Africa; ${ }^{2}$ Family Practitioner, Silverton, Pretoria, 0001, South Africa; ${ }^{3}$ Department of Pathology and Laboratory
Medicine and Emory Center for AIDS Research, Emory University, School of Medicine, Atlanta, GA 30322, United
States of America and ${ }^{4}$ Department of Medical Microbiology, University of Pretoria, Pretoria, 0001, South Africa

\begin{abstract}
Background: This study was undertaken to determine co-infection rates of common aetiological agents of urethritis and information regarding sexual contacts of symptomatic males presenting to a family practitioner.

Methods: Endo-urethral swab specimens were collected from 253 symptomatic men and tested for N. gonorrhoeae, C. trachomatis by the BD ProbeTec ${ }^{\mathrm{TM}} \mathrm{ET}$ assay and for $T$. vaginalis by real-time PCR. Information regarding sexual behaviour was collected by the practitioner using a structured interview.

Results: Of the 253 patients investigated, 50 complained of urethral discharge with/without burning on micturition (BOM), the remaining 203 only complained of BOM. N. gonorrhoeae was isolated from 45\%, C. trachomatis from $15 \%$ and T. vaginalis from 5.5\%. N. gonorrhoeae/C. trachomatis co-infection was diagnosed in 7.5\% and T. vaginalis was detected in a higher number of males presenting without visible discharge. Similar number of men reported sexual contact only with their wives compared to men having casual sexual contacts. There were fewer gonococcal infections in men reporting sexual contact with their wives alone compared to men reporting casual sexual encounters.

Conclusions: Treatment guidelines for men presenting to Primary Health Care facilities and Family Practitioners need to consider the evolving antimicrobial resistance profiles and the presence of mixed infections in symptomatic patients.
\end{abstract}

Key Words: Neisseria gonorrhoeae, Chlamydia trachomatis, Trichomonas vaginalis, urethritis.

\section{INTRODUCTION}

Adult male urethritis in South Africa is a common clinical condition with fairly large numbers of patients presenting to family practitioners and primary health care facilities [1]. The symptoms of this condition include urethral discharge and/or burning on micturition (BOM) and occasionally dysuria. The established sexually transmitted pathogens implicated in the aetiology of urethritis include Neisseria gonorrhoeae, Chlamydia trachomatis (D-K strains) and Trichomonas vaginalis whilst the pathogen status of Mycoplasma genitalium and Ureaplasma urealyticum is unclear $[2,3]$.

In South Africa, like many other developing countries, syndromic management of sexually transmitted infections is advocated and the National Department of Health formulated treatment guidelines in 1996 [4]. These guidelines are widely used by public health service providers working at Primary Health Clinics as well as private and family practitioners. The prevalence of co-infections with common aetiological agents of male urethritis as well as antimicrobial susceptibility profiles [5] informs therapeutic guidelines and especially syndromic management.

*Address correspondence to this author at the Department of Medical Microbiology, University of Pretoria, PO Box 2034, Pretoria, 0001 Pretoria, South Africa; Tel: +27 12319 2271; Fax: +27 12321 9456;

E-mail: anwar.hoosen@up.ac.za
In a recent study we reported the antimicrobial susceptibility profiles of gonococcal isolates. The local isolates were resistant to the commonly used therapeutic agents and we advocated a change in the national treatment guidelines for $N$. gonorrhoeae [5]. To determine the prevalence of coinfections in men with urethritis, molecular assays were used in view of the higher sensitivity of detection.

\section{METHODS}

Endo-urethral swab specimens were collected from 253 consecutive symptomatic men presenting with urethral discharge with/without BOM to a family practitioner in Pretoria, South Africa. The urethral swab specimens were stored in $1 \mathrm{ml}$ of sterile distilled water at $-70^{\circ} \mathrm{C}$ for batch testing. Information regarding sexual behaviour was collected by the practitioner (AA) using a structured interview. HIV status was not determined.

C. trachomatis and $N$. gonorrhoeae testing was performed using the BD ProbeTec TM ET assay (Becton Dickinson, Sparks MD, USA). $200 \mu$ l of the sample was used for testing according to the manufacturer's protocol. T. vaginalis testing was performed using a laboratory developed realtime PCR assay as previously described [6]. The target of the assay was a 67-base pair region of the repeated sequence of the T. vaginalis genome [7]. A clinical evaluation of the as- 
say showed the real-time PCR test detected about $40 \%$ more positive samples than culture [6].

Statistical analysis was performed using Fisher Exact Test to determine whether there was any relation between two categorical variables.

Approval to perform this study was obtained from the Medical Research and Ethics committee (MCREC) of the University of Limpopo. (MP130/2005).

\section{RESULTS}

Two hundred and fifty three (253) specimens were collected from consecutive, symptomatic male patients. Their ages ranged from 15 to 73 years, with the mean age of 37 years. Of the 253 specimens collected, 50 were from patients complaining of a urethral discharge with/without BOM. The remaining 203 patients complained only of BOM and there was no visible urethral discharge on examination.

The prevalence of single and mixed infections is shown in Fig. (1). N. gonorrhoeae was detected in a total of 113/253 (44.7\%), C. trachomatis in 38/253 (15.0\%) and $T$. vaginalis in $14 / 253(5.5 \%)$ of the men recruited for the study. Co-infection with $N$. gonorrhoeae and C. trachomatis was found in 19/253 (7.5\%); C. trachomatis and T. vaginalis in only $1 / 253(0.4 \%)$ of the study subjects. No patient was infected with all 3 pathogens. None of the 3 pathogens were detected in $108(42.7 \%)$ symptomatic men.

The breakdown of infections according to symptoms of only BOM or urethral discharge with/without BOM is shown in Fig. (2). In men who had a visible urethral discharge $N$.

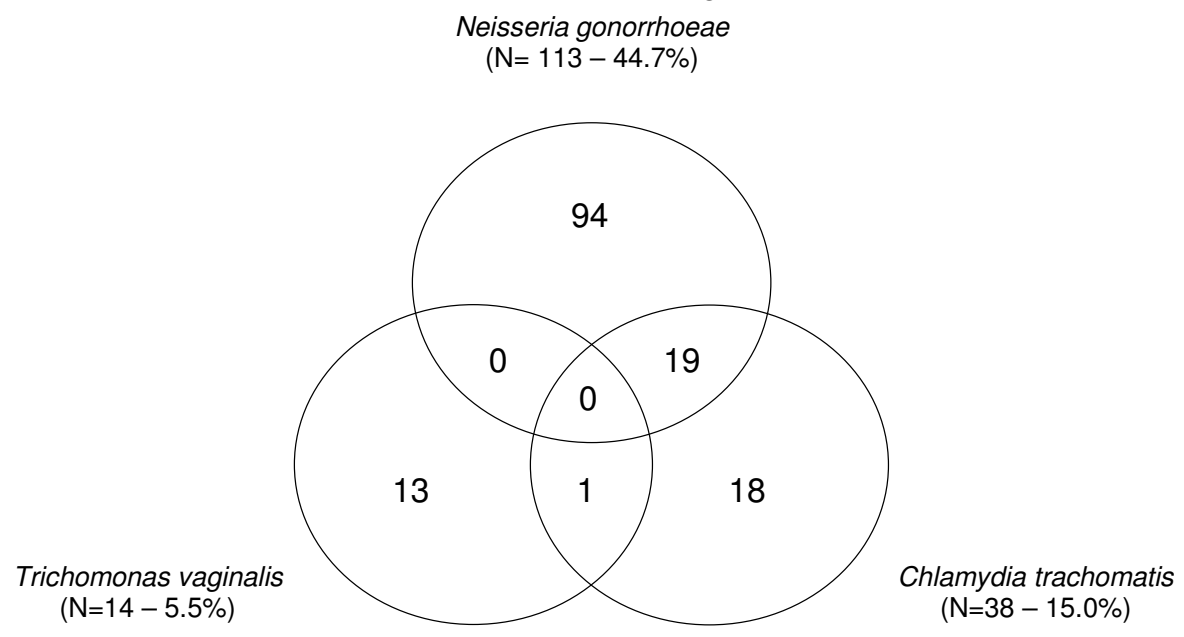

Fig. (1). Single and mixed infections in symptomatic men $(\mathrm{N}=253)$.

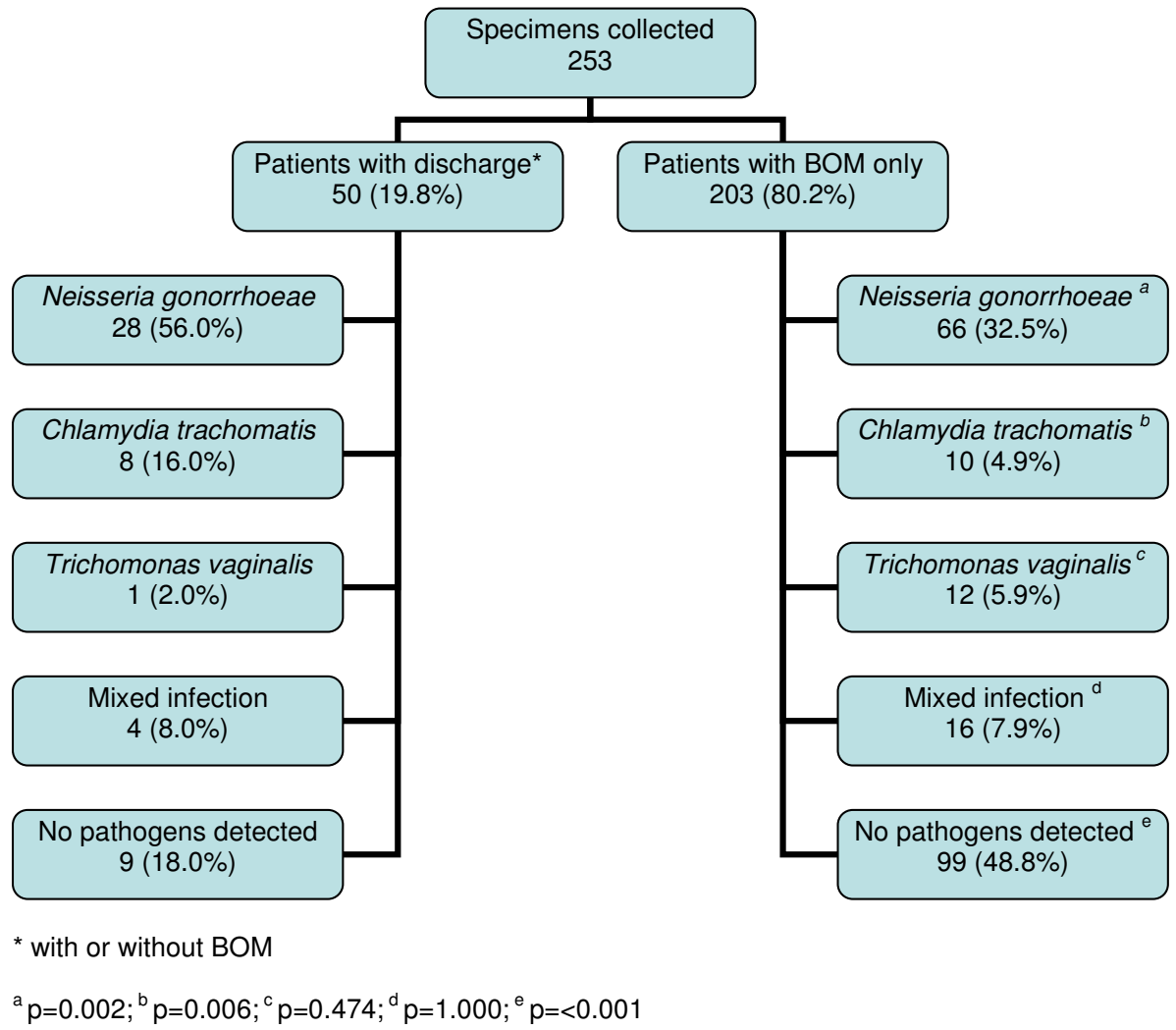

Fig. (2). Infections according to symptoms $(\mathrm{N}=253)$. 
gonorrhoeae was detected in $28(56.0 \%)$; C. trachomatis in $8(16.0 \%)$ and $T$. vaginalis infection in only one $(2.0 \%)$. In four $(8.0 \%)$ of the study subjects, co-infection with $N$. gonorrhoeae and $C$. trachomatis was found and in $9(18 \%)$ none of the pathogens was detected.

Of the men who complained only of BOM, in 99 (48.8\%) none of these pathogens were detected; $N$. gonorrhoeae was diagnosed in $66(32.5 \%)$, C. trachomatis in $10(4.9 \%)$ and $T$. vaginalis in $12(5.9 \%)$ of the men. $N$. gonorrhoeae and $C$. trachomatis co-infection was found in 15 (7.4\%) of the men and co-infection with $C$. trachomatis and $T$. vaginalis in only one $(0.5 \%)$ person. There were significant differences for $N$. gonorrhoeae $(56.0 \%$ vs $32.5 \% ; \mathrm{p}=0.002)$, C. trachomatis $(16.0 \%$ vs $4.9 \% ; \mathrm{p}=0.006)$ and where no pathogens were detected $(48.8 \%$ vs $18.0 \%$; $=<0.001)$ when comparing the groups.

A total of $54(21.3 \%)$ study subjects suffered from other illnesses such as diabetes and influenza (diagnosed clinically). Seven patients $(2.8 \%)$ were clinically diagnosed to have prostatitis, but no organisms were detected in the urethral specimens from these patients.

Regarding sexual behaviour, one hundred and fourteen $(45.1 \%)$ men reported their wives as the only contact, whilst $112(44.3 \%)$ of the men admitted to having sex with casual contacts (Table 1). Only one man (0.4\%) admitted to sexual contact with his wife as well as casual acquaintances, whilst $24(9.5 \%)$ of the men stated that they had steady relationships (with one partner) and for $2(0.8 \%)$ of the participants no information was available.

For the pathogens identified according to sexual contacts, there were significant differences only where no pathogen was isolated when comparing men who had sex with their wives as sole partners versus those who had sex with casual partners.

\section{DISCUSSION}

This study determined the co-infection rate of $N$. gonorrhoeae, $C$. trachomatis and $T$. vaginalis in adult males presenting with symptoms of urethritis to a family practitioner in the city of Pretoria, South Africa. The overall infection rate was $65 \%$ with the co-infection rate (i.e. more than one pathogen detected) of $8 \%$ (20 of 253). The infection rate in a similar study also using molecular diagnostic assays in a high HIV prevalence area of South Africa for the same three pathogens was $74 \%$ [3]. The similarity between these studies is reflected by the diagnosis of $N$. gonorrhoeae being $45 \%$ vs. $52 \%$; C. trachomatis $15 \%$ vs. $16 \%$ and $T$. vaginalis $6 \%$ in each study. It must be noted that the HIV status was not determined in our study. However, the study from KwaZulu Natal (KZN) reported a $45 \%$ HIV prevalence and the HIV status did not affect the prevalence of the urethral pathogens [3]. However, in the current study, we looked at signs and symptoms of men with urethritis and information on the sexual contacts of symptomatic males.

Gonococcal urethritis using the more sensitive molecular assay was diagnosed in $45 \%$ of the study participants, which is considerably less than the range of $64-96 \%$ reported in an earlier review of South African studies [1]. However, this lower prevalence has also been reported in more recent studies such as the one from KZN (52\%) [3] and a recent study form our centre in Pretoria (39\%) [5].

Chlamydial infection was found in $15 \%$ of the participants. This is very similar to what was found in the 1996 review by Pham-Kanter [1] where prevalences between 12 $14 \%$ were reported. This was in an era where laboratory testing for chlamydial infection was reliant on culture and antigen detection methods. It is also a striking similarity that using molecular techniques, the KZN study found a $16 \%$ rate of infection [3] and a more recent study from Johannesburg reported $19 \%$ [8]. When analyzing the type of symptoms, $N$. gonorrhoeae and $C$. trachomatis were detected from significantly higher number of individuals presenting with a visible urethral discharge $(56.0 \%$ and $16.0 \%)$ as compared to those presenting with only BOM (32.5\% and $4.9 \%)$.

There have not been many studies on the prevalence and role of $T$. vaginalis in adult male urethritis in South Africa. Our study showed a prevalence rate of $6 \%$ which compares favorably with the $6 \%$ found in KZN [3] and the 5\% in Johannesburg [8]. In all these recent studies molecular diagnostic assays have been used. In a study by Crewe-Brown [9], a prevalence of $4 \%$ was reported. It is important to note that this earlier study used conventional methods such as culture and microscopy for the diagnosis of trichomoniasis. However, in our study there was a difference in the detection of $T$. vaginalis in men without visible discharge and only complaining of BOM as compared to those in whom there was a visible discharge. This needs to be confirmed by

Table 1. Sexual Contacts of Participants and the Pathogens Detected $(\mathrm{N}=253)$

\begin{tabular}{|c|c|c|c|c|c|c|}
\hline $\begin{array}{c}\text { Contact/ } \\
\text { Partner }\end{array}$ & $\begin{array}{c}\text { No pathogen } \\
\text { Number (\%) }\end{array}$ & $\begin{array}{c}\text { N.gonorrhoeae } \\
\text { Number (\%) }\end{array}$ & $\begin{array}{c}\text { C. trachomatis } \\
\text { Number (\%) }\end{array}$ & $\begin{array}{c}\text { T. vaginalis } \\
\text { Number (\%) }\end{array}$ & $\begin{array}{c}\text { Mixed } \\
\text { Number (\%) }\end{array}$ \\
\hline \hline Wife & $60(52.6 \%)$ & $38(33.3 \%)$ & $5(4.4 \%)$ & $6(5.3 \%)$ & $5(4.4 \%)$ & $114(45.1 \%)$ \\
\hline Casual contact & $37(33.0 \%)^{1}$ & $50(44.6 \%)^{2}$ & $9(8.0 \%)^{3}$ & $6(5.4 \%)^{4}$ & $10(8.9 \%)^{5}$ & $112(44.3 \%)$ \\
\hline Steady partner & $9(37.5 \%)$ & $5(20.8 \%)$ & $4(16.7 \%)$ & $1(4.2 \%)$ & $5(20.8 \%)$ & $24(9.5 \%)$ \\
\hline Not recorded & $1(50 \%)$ & $1(50 \%)$ & 0 & 0 & 0 & $2(0.8 \%)$ \\
\hline Wife \& Casual & $1(100 \%)$ & 0 & 0 & 0 & $13(5.14 \%)$ & $20(7.91 \%)$ \\
\hline
\end{tabular}

${ }^{1} \mathrm{p}=0.003 ;{ }^{2} \mathrm{p}=0.081 ;{ }^{3} \mathrm{p}=0.255 ;{ }^{4} \mathrm{p}=0.975 ;{ }^{5} \mathrm{p}=0.170$. 
studying and following-up a larger cohort of patients as this may have implications for therapy. There may be a need to add an anti-trichomonicidal agent in men presenting with complains of BOM in the absence of visible discharge. Coinfection was detected in a total of 20 individuals; the majority being $N$. gonorrhoeae and $C$. trachomatis (19 persons) and only one with $C$. trachomatis and $T$. vaginalis.

The pathogen status of M. genitalium is unclear and no investigation for this organism was performed in the current study. In a review on urethritis in men, $M$. genitalium was implicated as the causal agent in $18-46 \%$ of men with urethritis [2] and local South African studies reported overall prevalence of 5\% [3] and 7-14\% [8]. For syndromic management the antibiotic used for treating chlamydial infection invariably covers the genital mycoplasmas and this may be a factor in this potential pathogen not being investigated in many epidemiolological studies for implementation of syndromic management guidelines. Furthermore, one has to use molecular diagnostic methods to diagnose $M$. genitalium as it takes a very long time to culture.

With regard to sexual behavior, differences were found when comparing sexual partners and pathogens isolated. Significantly fewer pathogens were detected in males that had their wives as sole contact, when comparing men who had sex with casual contacts, reflecting high sexual risk behavior.

More than ten years ago, in 1996 the National Department of Health of South Africa implemented guidelines for the syndromic management of sexually transmitted diseases (STDs). For urethritis (discharge with/without BOM), a two agent regimen to cover for gonorrhoea and chlamydia was advocated viz. ciprofloxacin plus doxycycline. In view of the results obtained in this study, where $T$. vaginalis was detected in a higher number of males presenting without a visible discharge, the question is raised whether metronidazole should be included in the syndromic regimens for patients presenting with BOM only, or whether it should only be administered to patients with persistent urethral symptoms. There needs to be further studies to answer these questions. However, it is heartening to note that metronidazole is still the drug of choice for the treatment of trichomoniasis, as most local isolates remain susceptible [10].

In a recent article [5], we advocated a change in the syndromic management of patients with urethritis from ciprofloxacin to ceftriaxone as local isolates have become resistant to the quinolones [11]. The drugs of choice for the treatment of chlamydial infection remain the tetracyclines (doxycycline) or azithromycin. Azithromycin is favoured because of the ease of administration of a single dose.

\section{LIMITATIONS OF THIS STUDY}

HIV status of participants was not determined.

\section{ACKNOWLEDGEMENTS}

The authors wish to thank Mrs Z Adam for assisting in the collection of specimens, Jessica Ingersoll and Tameka Bythwood for performing the molecular testing. This work was supported in part by the Emory Center for AIDS Research (P30 AI050409).

\section{ABBREVIATIONS}

$\begin{array}{ll}\text { BOM } & =\text { Burning on micturition } \\ \text { C. } \text { trachomatis } & =\text { Chlamydia trachomatis } \\ \text { M. genitalium } & =\text { Mycoplasma genitalium } \\ \text { N. gonorrhoeae } & =\text { Neisseria gonorrhoeae } \\ \text { PCR } & =\text { Polymerase chain reaction } \\ \text { STD's } & =\text { Sexually transmitted diseases } \\ T . \text { vaginalis } & =\text { Trichomonas vaginalis } \\ \text { USA } & =\text { United States of America }\end{array}$

\section{REFERENCES}

[1] Pham-Kanter GBT, Steinberg MH, Ballard RC. Sexually transmitted diseases in South Africa. Genitourin Med 1996; 72: 160-71.

[2] Ishihara S, Yasuda M, Ito S, Maedo S, Deguchi. Mycoplasma genitalium urethritis in men. Int J Antimicro Agents 2004; 245: S23-7.

[3] Sturm PDJ, Moodley P, Khan N, et al. Aetiology of male urethritis in patients recruited from a population with a high HIV prevalence. Int J Antimicrob Agents 2004; 245: S8-14.

[4] Department of National Health, Directorate HIV/AIDS and STDs. Protocols for the management of a person with sexually transmitted diseases (STD), South Africa. Protocol no. 1 1996, Pretoria: DOH.

[5] De Jongh M, Dangor Y, Adam A, Hoosen AA. Gonococcal resistance: evolving from penicillin, tetracycline to the quinolones in South Africa - implications for treatment guidelines. Int J STD AIDS 2007; 18: 697-9.

[6] Caliendo AM, Jordan JA, Green, AM, Ingersoll J, Wingood GM, DiClemente RJ. Real-Time PCR provides improved detection of Trichomonas vaginalis infection compared to culture using selfcollected vaginal swabs. Infect Dis Obstet Gynecol 2005; 13: 145 50 .

[7] Kegne P, Veas F, Vidal N, Rey JL, Cuny G. Trichomonas vaginalis: repeated DNA target for highly sensitive and specific polymerase chain reaction diagnosis. Cell Mol Biol 1994; 40: 819-31.

[8] Black V, Magooa P, Radebe F, Myers M, Pillay C, Lewis DA. The detection of urethritis pathogens among patients with the male urethritis syndrome, genital ulcer syndrome and HIV VCT clients: Should South Africa's syndromic management approach be revised? Sex Transm Infect 2008; 84: 254-8.

[9] Crewe-Brown HH, Adam A, Ebrahim O, Mahomed MF, Pochee E. The aetiology of acute urethritis in a southern African general practice. South Afr J Epidemiol Infect 1991; 6: 31-3

[10] Fernandes L, Garvey SB, Hoosen AA. In-vitro susceptibility testing of Trichomonas vaginalis. International Society for Sexually Transmitted Diseases Research Congress, Ottawa, Canada, July 2003; 27-30.

[11] Koornhof HJ, Moodley P, Slabbert M, et al. A national survey of antimicrobial resistance in gonococci isolated in South Africa. $1^{\text {st }}$ Joint Congress of the Federation of Infectious Diseases Societies of southern Africa, abstract OR1. 